\title{
EL ABUSO DE AUTORIDAD DOCENTE: DESARROLLO DE UN INSTRUMENTO DE MEDIDA
}

\section{TEACHER'S ABUSE OF AUTHORITY: DEVELOPMENT OF A MEASURING INSTRUMENT}

\author{
Marta Hernández Arriaza \\ María Prieto Ursúa ${ }^{1}$ \\ Universidad Pontificia Comillas, España
}

\section{RESUMEN}

Actualmente no existen antecedentes de herramientas robustas de evaluación del Abuso de Autoridad Docente en el contexto español. El objetivo del estudio es presentar el proceso de desarrollo de la Escala de Abuso de Autoridad Docente Percibido (EAADP) así como plantear un modelo de medida que permita estructurar teóricamente la escala. La escala fue aplicada a una muestra de 306 estudiantes de Madrid capital con edades comprendidas entre los 14 y 18 años. Los resultados muestran que el instrumento obtiene adecuados valores de consistencia interna y el análisis factorial sustenta la estructura multidimensional propuesta para la escala. El modelo final consta de cuatro factores de primer orden, o estilos de abuso (distante, degradante, sancionador y arbitrario), y una variable latente denominada Abuso de Autoridad Docente (AAD). Además, el AAD correlaciona negativamente con el bienestar personal y académico de los alumnos. La EAADP permite medir con validez y fiabilidad el abuso de autoridad docente percibido por los alumnos a lo largo de su escolaridad.

Palabras clave: Abuso docente, autoridad docente, clima escolar, abuso escolar

\footnotetext{
${ }^{1}$ Correspondencia: Marta Hernández Arriaza: Universidad Pontificia Comillas - C/ Alberto Aguilera 23 - 28015 Madrid. Correo-e: marta hdez@alu.comillas.edu ,web: http://www.comillas.edu/es/
} 


\section{ABSTRACT}

This study focuses on the unfair treatment some teachers have with their students, abusing their authority and consequences on personal and academic welfare. Currently there is no history of robust assessment tools of Teacher's Abuse of Authority in the Spanish context. The aim of the study is to present the development process of the Scale of Perceived Teacher Abuse of Authority (EAADP) and propose a model to structure theoretically the scale. The scale was applied to a sample of 306 students in Madrid, aged between 14 and 18 years. The results show that the instrument obtains adequate values of internal consistency and factor analysis supports the proposal for the scale multidimensional structure. The final model consists of four first-order factors or styles of abuse (distant, degrading, punitive and arbitrary), and a latent variable named Teacher Abuse of Authority (AAD). In addition, the AAD negatively correlated with personal and academic wellbeing of students. EAADP measures with validity and reliability the teacher abuse of authority perceived by students throughout their schooling.

Keywords: bullying by teachers, school bullying, aggression, classroom management

\section{Introducción}

Existe evidencia del impacto de las relaciones entre el profesorado y el alumnado de un aula (Bacete, Coll, Casares, y Perrin, 2014) y la buena calidad de las relaciones entre profesores y alumnos se relaciona con una mejor respuesta a las necesidades de desarrollo de los niños (Buyse, Verschueren, Doumen, Van Damme, y Maes, 2008). Cuando los profesores carecen de los recursos o estrategias que les permiten manejar de forma eficaz los desafíos sociales y emocionales dentro de la clase, el clima del aula se deteriora, y aumentan los conflictos con los alumnos (Mantilla y Díaz, 2015). De este modo, aunque los profesores sean las personas que deban velar por el bienestar de los estudiantes y modelar su aprendizaje proporcionándoles seguridad y confianza (McEachern, Aluede y Kenny, 2008), en algunas situaciones, el rol del profesor como agente protector no se cumple y se dan abusos de poder y otras formas de maltrato psicológico que afectan al bienestar emocional del alumno.

Las experiencias de abuso emocional o psicológico por parte de un profesor han sido tradicionalmente poco estudiadas en comparación con otros conflictos escolares. Sin embargo en los últimos años el abuso de autoridad docente ha cobrado interés en otros países entre los que se encuentran Estados Unidos (Sharpe, 2012; Boyle, 2013), Canadá (Brendgen, Bukowski, Wanne, Vitaro, y Tremblay, 2007), Yemen (Ba-Saddik, y Hattab, 2012), India (Baluran, y Yap, 2013), y Noruega (Monsvold, Bendixen, Hagen, y Helvik, 2011). Existe cierta discrepancia en la literatura internacional a la hora de nombrar este constructo, encontrando expresiones como abuso emocional docente (Ba-Saddik, y Hattab, 2012), maltrato psicológico (Lyles, 2014), bullying docente (Twemlow y Fonagy, 2005; Whitted y Dupper, 2008; Sylvester, 2010) o abrasive teachers (Weller, 2014). Teniendo en cuenta esta discrepancia, en este estudio tomamos como referencia la definición propuesta por Davies (2011) del término bullying docente, que hemos traducido como abuso de autoridad docente, recalcando la posición de autoridad que ostenta el profesor dentro del aula. Esta definición pone énfasis en el desequilibrio que se produce cuando el profesor utiliza su posición de poder de forma abusiva para castigar, manipular o denigrar a un estudiante causando un sufrimiento emocional considerable que va más allá de los métodos disciplinarios que se entienden como razonables.

El papel de la autoridad y el poder del profesor es fundamental para entender el abuso de autoridad docente. Así, los profesores ejercen tres tipos de poder sobre sus estudiantes debido a la estructura escolar: (1) El poder de experto por el que los profesores tienen el conocimiento que 
los estudiantes necesitan; (2) El poder formal por el que los profesores tienen el derecho de estructurar el tiempo y las actividades de los estudiantes; y (3) el poder de los recursos con el que los profesores pueden determinar el comportamiento de un alumno en función de lo que se refuerce o no a un niño en particular (Mullet, 2006). El abuso de autoridad docente está formado por una serie de conductas que pueden agruparse en función de sus consecuencias para el alumno. Nesbit y Philpott (2002) encontraron en su análisis factorial seis tipos de conductas que componen el abuso emocional docente: (1) Las conductas degradantes que humillan, denigran y disminuyen la autoestima mediante el bochorno y el desprecio verbal; (2) Las conductas discriminantes que perjudican a individuos o grupos basándose en su sexo, raza, nivel económico o habilidades cognitivas; (3) las conductas dominantes que definen parámetros de interacción y control, limitan o reprimen el crecimiento en competencia, confianza o pensamiento crítico; (4) las conductas desestabilizadoras que intimidan o producen ansiedad, miedo o tensión; (5) las conductas distanciantes que indican rechazo, aislamiento y falta de apoyo emocional, crean inseguridad a través de la insensibilidad hacia los sentimientos y la indiferencia hacia las necesidades emocionales; y (6) las conductas diversas que se relacionan con las actitudes del profesor que influyen negativamente en el ambiente de clase. Aunque no existen apenas estudios sobre el abuso de autoridad docente en nuestro país, en el ámbito internacional se ha indicado que el $88 \%$ de los alumnos informaron haber sufrido al menos un incidente de maltrato psicológico por parte de un docente (Whitted y Dupper, 2008) y el $45 \%$ de los profesores admitieron haber acosado a un estudiante (Twemlow, Fonagy, Sacco, y Brethour, 2006). Parece ser que cualquier persona puede ser víctima de acoso en un momento de su vida con independencia de su estatus socioeconómico, raza, edad, sexo orientación sexual o posición (Davies, 2011).

El abuso de autoridad docente comparte algunas características con el bullying entre alumnos. Como este último, el docente, es un tipo de abuso en el que se percibe un desequilibrio de poder, a favor del docente, tiende a ser repetitivo y a menudo tiene lugar en público (Twemlow et al., 2006). Del mismo modo que en el bullying entre alumnos, el profesor que abusa de su autoridad normalmente no recibe castigo o sanción (McEvoy, 2005).

Por último, como en el bullying entre alumnos las consecuencias del abuso de autoridad docente van más allá del ámbito académico y escolar. Aquellos estudiantes que son acosados por un profesor suelen experimentar confusión, miedo, enfado, desconfianza en sí mismos, y una profunda preocupación por su competencia social y académica. No saber por qué él o ella han sido escogidos, o qué deben hacer para terminar con el acoso puede ser uno de los aspectos personales más estresantes. Con el paso del tiempo, especialmente si ninguna figura de autoridad interviene, el alumno puede comenzar a culparse a sí mismo por el abuso y por consiguiente tener un sentimiento generalizado de inutilidad y desamparo. Las consecuencias del abuso de autoridad docente afectan a varias facetas de la vida del alumno, enmascarándose en ocasiones mediante problemas de conducta y escolares (Lyles, 2014) o interacciones negativas con los profesores que pueden convertirse en personas temibles de las que hay que escapar (McEachern et al., 2008). El alumno puede volverse frio, insensible o duro y puede experimentar ansiedad y fobia hacia la escuela. El abuso emocional por parte del profesor puede afectar al desarrollo cognitivo del alumno e incluso llevarle a abandonar la escuela por falta de motivación o miedo (Ceja et al., 2011). Además, esta experiencia puede influir en el establecimiento de futuras relaciones o a la expresión de sus emociones, al resultarle difícil confiar en otras personas (Davies, 2011). Estos alumnos se caracterizan por un comportamiento desconfiado, hipervigilante y con la apariencia de estar siempre a la defensiva. El abuso emocional suele dar lugar a baja autoestima, problemas de sueño, depresión, o síntomas físicos como dolores de cabeza o estómago (McEachern et al., 2008). Como resultado, las víctimas de acoso docente pueden sufrir consecuencias a largo plazo que afecten a su felicidad y éxito futuro (Davies, 2011).

Existen diversas teorías para estudiar el abuso de autoridad docente. Algunas estudian las relaciones entre profesores y alumnos basándose en la teoría del apego (Pianta, Hamre y Stuhlman, 2003) y centrándose en el profesor como figura con la que el niño puede establecer un 
vínculo significativo. Otras abordan investigaciones desde la teoría de la motivación social que sugiere que la función de un profesor es dar respuesta a las necesidades básicas de pertenencia, competencia y autonomía del alumno (Bacete et al., 2014). Fraire, Longobardi, Prino, Sclavo y Settanni (2013) utilizan la teoría sistémica añadiendo una visión contextual al problema.

La teoría de la desconexión moral de Bandura, Barbarenelli, Caprara y Pastorelli (1996) afirma que las personas hemos desarrollado estándares morales que rigen la vida y comportamiento diario mediante procesos de autocensura moral. No obstante, estos mecanismos pueden desactivarse para ciertas conductas, permitiendo que se lleven a cabo acciones moralmente reprochables sin consecuencias negativas para el que las realiza. Es decir, existen en nuestro sistema cognitivo una serie de mecanismos que permiten al ser humano actuar de forma contraria a sus valores sin que se activen sentimientos negativos como la culpa (Bandura, 2002). En ocasiones, los profesores utilizan los mecanismos de desconexión moral para justificar las malas acciones educativas (Torre Puente, 2016).

En España la literatura presente hasta la fecha se ha limitado, en su mayoría, a la validación de cuestionarios en castellano para medir la calidad de la relación profesor alumno (Torres, Infante, Mena, De la Morena, Raya, y Muñoz, 2006; Bacete et al., 2014). Incluso, se ha realizado un estudio a nivel estatal sobre la convivencia escolar en la Educación Secundaria (Díaz-Aguado, Martínez Arias, y Martín, 2010). Sin embargo, estos estudios no contienen ítems concretos sobre el abuso de autoridad docente. Esta situación se debe a que tal y como refieren Ceja, Cervantes y Ramírez (2011) el tema de la violencia que el maestro ejerce sobre sus alumnos parece estar vetado al estudio y a la investigación; es un tema tabú del que no se habla y apenas existen investigaciones. Existen algunos cuestionarios fuera de nuestro país, entre los que merece la pena destacar el Teacher Bullying Behaviors Questionnaire (Davies, 2011) y el cuestionario de Ba-Saddik y Hattab (2012) desde la perspectiva del alumno, el cuestionario de Twemlow et al. (2006) desde la del profesor, así como la versión de Lyles, (2014) desde la perspectiva del orientador. No existen, sin embargo, cuestionarios o escalas en castellano que midan específicamente el abuso de autoridad docente percibido por el alumno y por tanto, hasta ahora no existen datos sobre la prevalencia de este fenómeno en la población española.

Teniendo en cuenta la escasa investigación sobre el abuso de autoridad docente en España así como las consecuencias que este tipo de abuso tiene en el alumnado, es necesario contar con una herramienta de evaluación en castellano sustentada en un modelo teórico que permita explicar mejor el constructo medido. De esta manera, los objetivos de esta investigación son:

- Proporcionar un instrumento útil para su empleo en la evaluación y en la intervención del abuso de autoridad docente percibido por los alumnos.

- Evaluar la prevalencia, en muestra española, de alumnos que informan haber sufrido abuso de autoridad docente durante su escolaridad.

- Proponer un modelo de medida del abuso de autoridad docente.

- Relacionar las puntuaciones en abuso de autoridad docente con las puntuaciones en bienestar personal subjetivo.

\section{Método}

\section{Muestra}

El estudio se llevó a cabo con una muestra incidental de carácter no probabilístico de 306 alumnos de tercero y cuarto curso de Educación Secundaria Obligatoria procedente de tres 
centros de la Comunidad de Madrid. De los tres centros, dos eran de titularidad privadoconcertada y uno de titularidad pública. Del total de la muestra 232 alumnos $(75,81 \%)$ estaban cursando $3^{\circ}$ de ESO y los 74 restantes (23,78\%) cursaban 4을 tenían 14 años, el 42, 91\% 15 años, el 18,39\% 16 años, el 9,20\% 17 años y el 0,96\% tenían 18 años. Además, el $54,9 \%$ eran chicos y el $44,8 \%$ chicas.

\section{Instrumentos}

Para evaluar el abuso de autoridad docente se propuso la Escala de Abuso de Autoridad Docente Percibido, de elaboración propia. La elaboración de la EAADP se desarrollótomando como muestra el cuestionario de Davies (2011) y Lyles (2014) y siguiendo la clasificación propuesta por Nesbit y Philpot (2002) que engloba las conductas abusivas dentro de seis factores: degradante, discriminante, dominante, desestabilizadora, distanciante y diversa. Esta versión inicial de la escala estaba compuesta por 45 ítems que se valoran en una escala de tipo Likert de 5 puntos en las que 1 significa nunca y 5 siempre. El cuestionario que recoge la escala comienza con una situación estimular que ejemplifica el constructo de Abuso de Autoridad Docente. Los ítems se redactaron siguiendo el siguiente ejemplo: "El profesor/a se reía de mí delante de mis compañeros cuando sacaba una mala nota". Una vez redactados los ítems, se añadieron la situación estimular y los ítems de control. Esta primera versión del cuestionario fue validada por doce jueces. Los resultados fueron analizados desde el punto de vista cualitativo y cuantitativo, se eliminaron seis ítems por no ajustarse a la definición del constructo y se realizaron los cambios propuestos por los expertos, resultando un cuestionario de 39 ítems.

Para evaluar las consecuencias del abuso de autoridad en los alumnos, decidimos medir el Bienestar Personal Subjetivo (BPS), que se refiere a lo que las personas piensan y sienten acerca de sus vidas y a las conclusiones cognoscitivas y afectivas que ellos alcanzan cuando evalúan su existencia. Lo central es entonces, la propia evaluación que la persona hace de su vida (Diener, 2000). Esta variable se midió con la Escala de Satisfacción con la Vida en Adolescentes (Diener, Emmons, Larsen, y Griffin, 1985) validada en español por Atienza, Pons, Balaguer y García-Merita (2000), y compuesta por seis ítems tipo Likert ( $1=$ totalmente en desacuerdo; $5=$ totalmente de acuerdo). Un ejemplo de ítem es: "Estoy satisfecho con mi vida". Además, se incluyó la siguiente pregunta: "¿Alguna vez has sentido que un profesor te trataba injustamente abusando de su autoridad?". Las respuestas a esta pregunta oscilaban entre nunca y casi siempre; siendo 1= nunca y $5=$ casi siempre.

\section{Procedimiento}

La selección de los colegios se realizó en función del interés mostrado por sus equipos directivos a la realización de un proyecto de investigación sobre el abuso de autoridad docente. Se redactó una carta a los directores de los centros y se pidió su consentimiento informado. Una vez recogida la autorización por parte de los directores, se aplicó un piloto con 20 alumnos de uno de los centros. Se seleccionó a alumnos escolarizados en diversificación por su bajo nivel de comprensión lectora. El objetivo de este piloto fue observar fundamentalmente la comprensión de los ítems de la escala. Aunque los alumnos comprendieron las instrucciones y la redacción de los ítems era correcta, tras la aplicación de este piloto se incluyeron seis ítems con puntuación inversa para controlar la sinceridad de la escala: ítems 9, 18, 23, 37, 44, 50.

Los estudiantes cumplimentaron voluntariamente, y de forma anónima, el cuestionario colectivo en una hora de tutoría. Las pruebas fueron administradas tras una breve introducción sobre la finalidad de la prueba. Las instrucciones para contestar el cuestionario se explicaron oralmente antes de la cumplimentación, además de ser entregadas por escrito a los alumnos. Se les recordó que, si en algún momento sentían malestar al contestar las preguntas, se pusieran en contacto con la encuestadora. 


\section{Análisis de datos}

Una vez recogidas todas las escalas y codificados los datos se procedió a realizar análisis descriptivos, análisis factorial confirmatorio (AFC) de la estructura propuesta por el análisis factorial exploratorio, análisis de fiabilidad. Para confirmar la estructura subyacente entre los constructos propuestos, se realizó un análisis causal utilizando un modelo de ecuaciones estructurales. Aunque todos los análisis estadísticos se realizaron con IBM SPSS Statistics 21, se utilizó la versión 6.2 del programa EQS para la realización del modelo de ecuaciones estructurales.

Los criterios de bondad de ajuste utilizados fueron: Chi cuadrado entre los grados de libertad $\left(X^{2} / g l\right)$, Comparative Fit Index (CFI), McDonald Fit Index (MFI), Bentler-Bonett Nonnormed Fit Index (NNFI), Root Mean Squared Residual (RMR) y Root Mean- Square error ofaproximation (RMSEA). En este estudio se trabajó con un nivel de confianza del 95\%. Para valorar la relación entre dos variables cuantitativas como por ejemplo puntuación en Bienestar Personal Subjetivo y puntuación en AADP se utilizó la correlación de Pearson.

\section{Resultados}

Los datos descriptivos se recogen en la tabla 1. Tras los análisis de fiabilidad y validez decidimos eliminar los ítems $6,13,35,36$ y 46 por su poca contribución a la fiabilidad y los ítems $11,17,21,24,25,28,31,33,34,38,40,41,42,43,47,48$ y 49 por su poca representación al realizar el análisis factorial. El Alfa de Cronbach antes de eliminar los ítems era de 0,94 y la versión final de 15 ítems tiene una fiabilidad de 0,87. El análisis factorial exploratorio agrupa los ítems en 4 factores que explican el $61,10 \%$ de la varianza.

TABLA 1. Estadísticos descriptivos y correlación entre factores

\begin{tabular}{lccccccc}
\hline & $M$ & $D T$ & Factor 1 & Factor 2 & Factor 3 & Factor 4 & Total escala \\
\hline Factor 1 & 7,91 & 3,70 & 1 &, 47 &, 49 &, 56 &, 82 \\
Factor 2 & 5,50 & 2,43 & & 1 &, 45 &, 49 &, 73 \\
Factor 3 & 8,53 & 3,50 & & & 1 &, 47 &, 79 \\
Factor 4 & 5,88 & 2,80 & & & & 1 &, 77 \\
Total escala & 27,82 & 9,79 & & & & & 1 \\
\hline
\end{tabular}

La escala final está compuesta por 4 factores:

- Factor 1: Estilo distante. Está formado por los ítems 16, 20, 22 y 30. Este factor explica el $15,89 \%$ de la varianza de la escala total. Su alfa es aceptable (alfa de 0,71). Además, el análisis factorial de este factor revela que explican el $55,28 \%$ de la varianza. Se trata de un factor unidimensional.

- Factor 2: Estilo degradante. Este factor está compuesto por los ítems 12, 1415 y 19 y explican el $15,23 \%$ de la varianza de la escala total. La fiabilidad de este factor es aceptable (alfa de 0,76). Se trata de un factor unidimensional cuyos ítems explican el $58,821 \%$ de la varianza. 
- Factor 3: Estilo sancionador. Está formado por los ítems 27, 29, 32 y 45 . Este factor explica el $15,23 \%$ de la varianza de la escala total. Su fiabilidad es aceptable (alfa de 0,71): Además, el análisis factorial de este factor revela que explican el 58,64\% de la varianza. Se trata de un factor unidimensional.

- Factor 4: Estilo arbitrario. Este factor está compuesto por los ítems 7, 8 y 10. Estos ítems explican el $14,24 \%$ de la varianza de la escala total. La fiabilidad de este factor es aceptable (alfa de 0,76). Se trata de un factor unidimensional cuyos ítems explican el $68,18 \%$ de la varianza.

\section{TABLA 2. Matriz de componentes rotados}

\begin{tabular}{|c|c|c|c|}
\hline \multirow{4}{*}{ 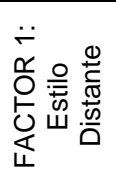 } & 16. Evitaba preguntarme en clase & ,678 & \multirow{4}{*}{$\begin{array}{c}\text { Alfa de } \\
\text { Cronbach=0,71 } \\
\text { Var. Total }=15,89 \%\end{array}$} \\
\hline & 20. Me ignoraba fuera de clase (patios, pasillos, despacho...) & ,698 & \\
\hline & 22. Ignoraba mis preguntas en clase & ,675 & \\
\hline & 39. Cuando yo lograba algo, el/ ella evitaba felicitarme & ,699 & \\
\hline \multirow{4}{*}{ 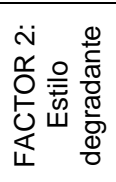 } & 12. Se reía de mí delante de mis compañeros cuando sacaba una mala nota & ,708 & \multirow{4}{*}{$\begin{array}{c}\text { Alfa de } \\
\text { Cronbach }=0,75 \\
\text { Var. Total }=15,74 \%\end{array}$} \\
\hline & 14. Se burlaba de mi ropa o peinado & ,738 & \\
\hline & 15. Me ridiculizaba delante de mis compañeros & 690 & \\
\hline & 19. Hacía comentarios inapropiados sobre mi situación familiar & ,722 & \\
\hline \multirow{4}{*}{ 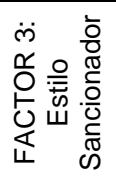 } & 27. Me castigaba por infracciones insignificantes & ,680 & \multirow{4}{*}{$\begin{array}{c}\text { Alfa de } \\
\text { Cronbach }=0,76 \\
\text { Var. Total }=15,23 \%\end{array}$} \\
\hline & 29. Me gritaba en clase & ,642 & \\
\hline & 32. Castigaba a toda la clase cuando me portaba mal & ,789 & \\
\hline & 45. Me castigaba separándome del resto de la clase & ,722 & \\
\hline \multirow{3}{*}{ 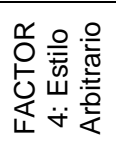 } & 7. Me daba la sensación de que evaluaba mis trabajos o exámenes sin leerlos & ,684 & \multirow{3}{*}{$\begin{array}{c}\text { Alfa de } \\
\text { Cronbach }=0,76 \\
\text { Var. Total }=14,24 \%\end{array}$} \\
\hline & 8. Corregía mis exámenes con un rigor excesivo para suspenderme & ,780 & \\
\hline & 10. Me suspendía sin tener en cuenta mi esfuerzo o mis progresos & ,785 & \\
\hline
\end{tabular}

Esta estructura factorial nos sugiere tres posibles explicaciones. La primera explicación es que exista un solo factor unidimensional llamado abuso de autoridad docente. La segunda explicación es que haya 4 factores (estilos de abuso) que correlacionen entre sí. Por último, el tercer modelo propone la existencia de cuatro factores de primer orden y un factor de segundo orden.

Una vez completada la aproximación exploratoria de datos, pasamos a análisis más complejos que permiten probar estadísticamente la validez de las ecuaciones estructurales. La estimación de los modelos se llevó a cabo mediante el método de máxima verosimilitud, utilizado cuando es posible asumir normalidad multivariada. En nuestro caso en ninguno de los modelos el Coeficiente de Mardia (curtosis multivariada) superó el criterio $p \times(p+2)$ donde $p$ se refiere al número de variables observadas. En nuestro caso ningún modelo superó el valor 255: $15 \mathrm{x}$ $(15+2)$. El valor del Coeficiente de Mardia para los tres modelos es de 202,34.

TABLA 3. Índices de bondad de ajuste de los 3 modelos

\begin{tabular}{|c|c|c|c|c|c|c|c|c|c|}
\hline Modelo & $x^{2}$ & $g l$ & $x^{2} / g l$ & GFI & AGFI & $C F I$ & $N F I$ & SRMR & RMSEA \\
\hline Modelo de 1 sólo factor & 366,44 & 90 & 4,04 & 0,84 & 0,79 & 0,81 & 0,77 & 0,08 & 0,10 \\
\hline $\begin{array}{l}\text { Modelo de } 4 \text { factores } \\
\text { correlacionados }\end{array}$ & 111,96 & 84 & 1,33 & 0,95 & 0,94 & 0,98 & 0,97 & 0,04 & 0,03 \\
\hline $\begin{array}{l}\text { Modelo de } 4 \text { factores de } \\
\text { primer orden y } 1 \text { de segundo } \\
\text { orden }\end{array}$ & 112,55 & 85 & 1,32 & 0,95 & 0,94 & 0,98 & 0,97 & 0,04 & 0,03 \\
\hline
\end{tabular}


Como observamos en la tabla 3, el ajuste del Modelo Inicial de Medida (Figura 1) en el que se incluyeron los 15 indicadores que forman el Abuso de Autoridad Docente medido por nuestra escala es pobre. El valor de los residuos estandarizados es superior al valor absoluto de 0,15, llegando al 0,20.

Basándonos en el AFE realizado incluimos cuatro factores de primer orden con el fin de comprobar si se producía una mejora en el ajuste del modelo. El segundo modelo o Modelo de medida de 4 factores interrelacionados, representado en la figura 2, presenta un buen ajuste: el valor de Chi cuadrado disminuye, siendo de 111,96; $x^{2} / g l$ es menor que $3(1,33)$; el GFI $(0,95)$, AGFI $(0,94)$, CFI $(0,98)$, y NNFI $(0,97)$ son superiores a 0,90 , el SRMR es próximo a $0(0,04)$, y el RMSEA $(0,03)$ es inferior a 0,08 .

FIGURA 1. Modelo de medida de un solo factor.

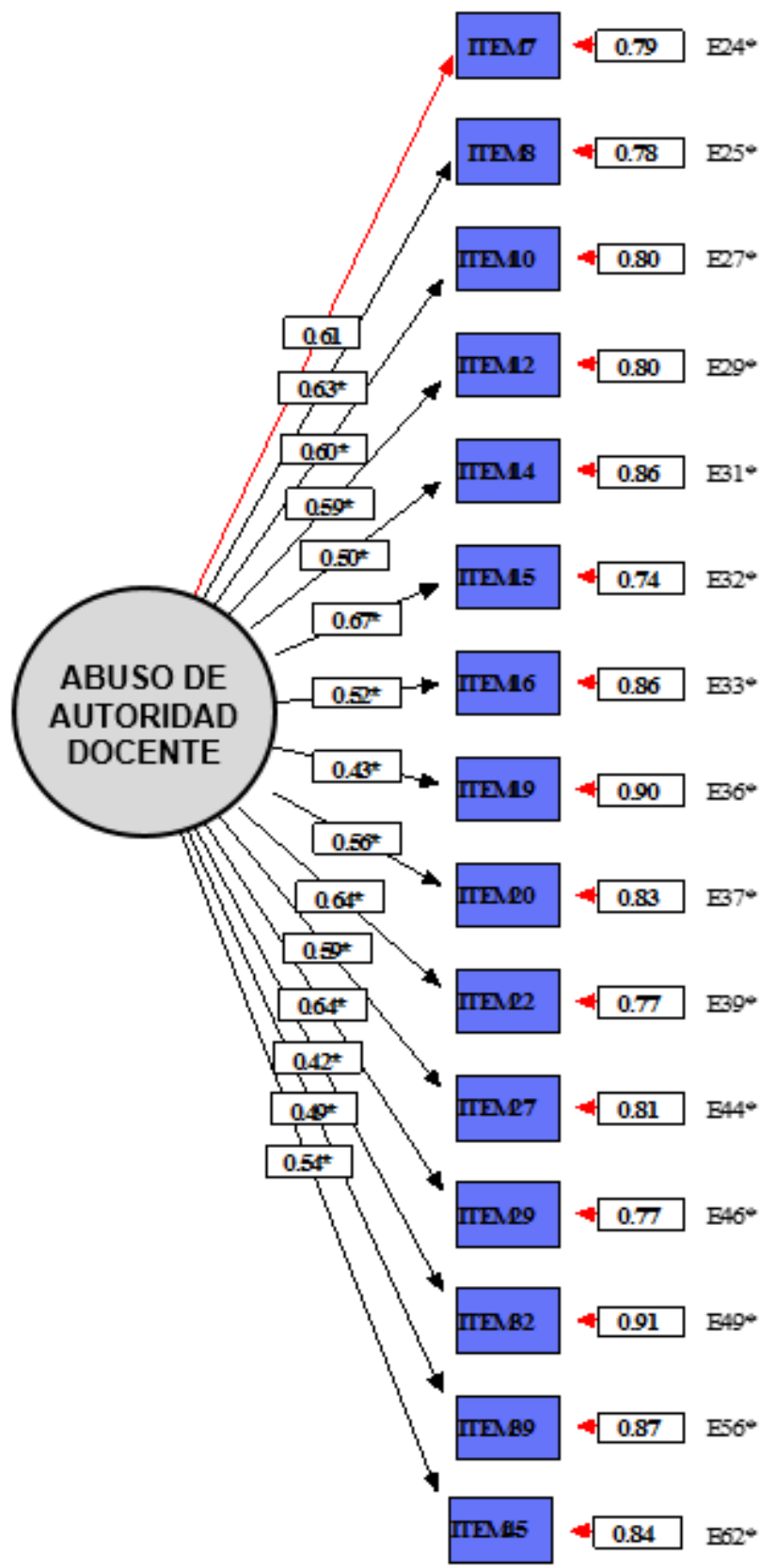


FIGURA 2. Modelo de medida de cuatro factores interrelacionados

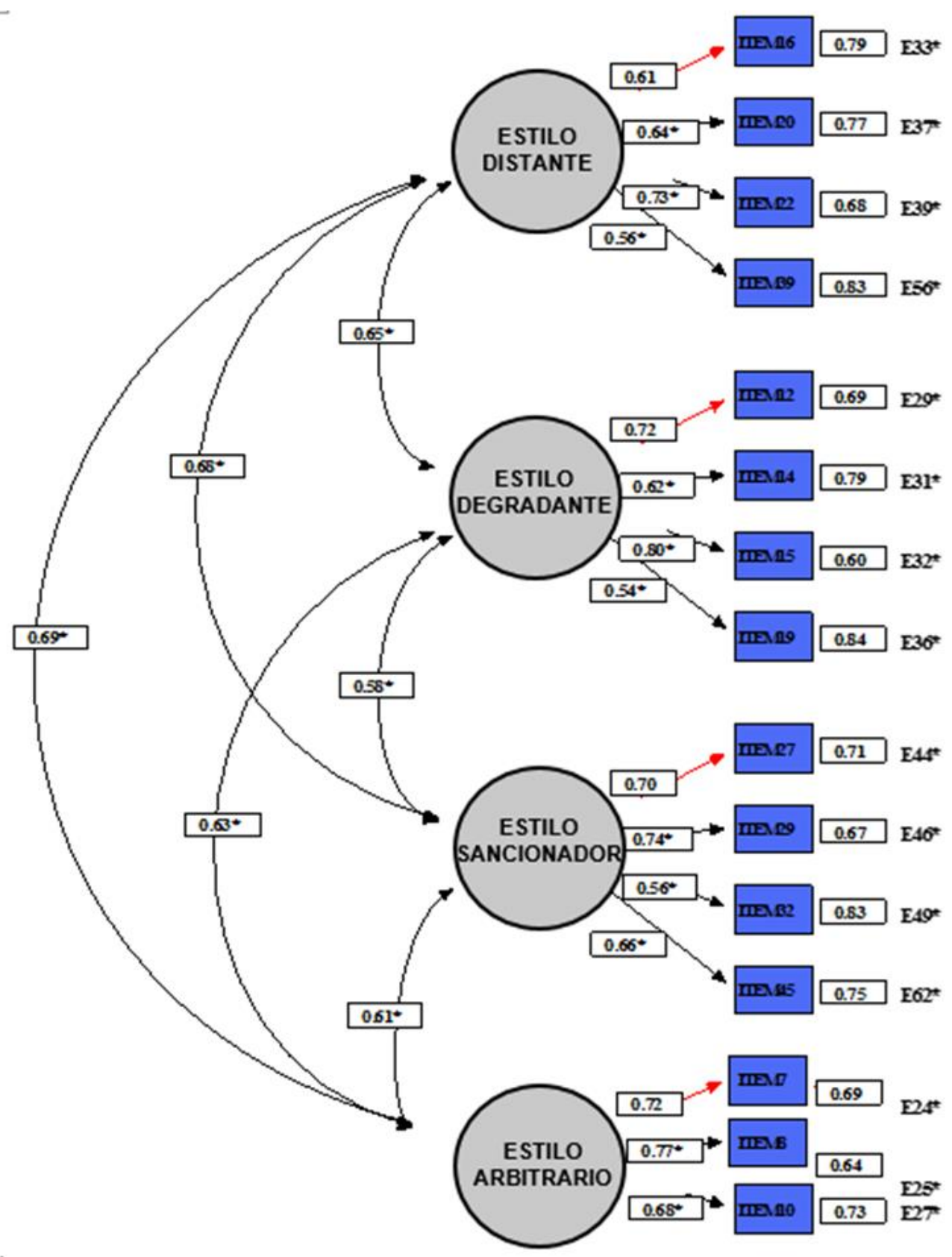

Aunque la bondad de ajuste del segundo modelo es muy robusta, la teoría nos dice que es posible que exista un factor latente a estas cuatro dimensiones: el abuso de autoridad docente. En la figura 3 aparecen representados 15 indicadores (variables manifiestas), agrupadas en 4 variables de primer orden (distante, degradante, sancionador y arbitrario) que permiten estimar la variable latente abuso de autoridad docente. 
FIGURA 3. Modelo de medida con una variable latente

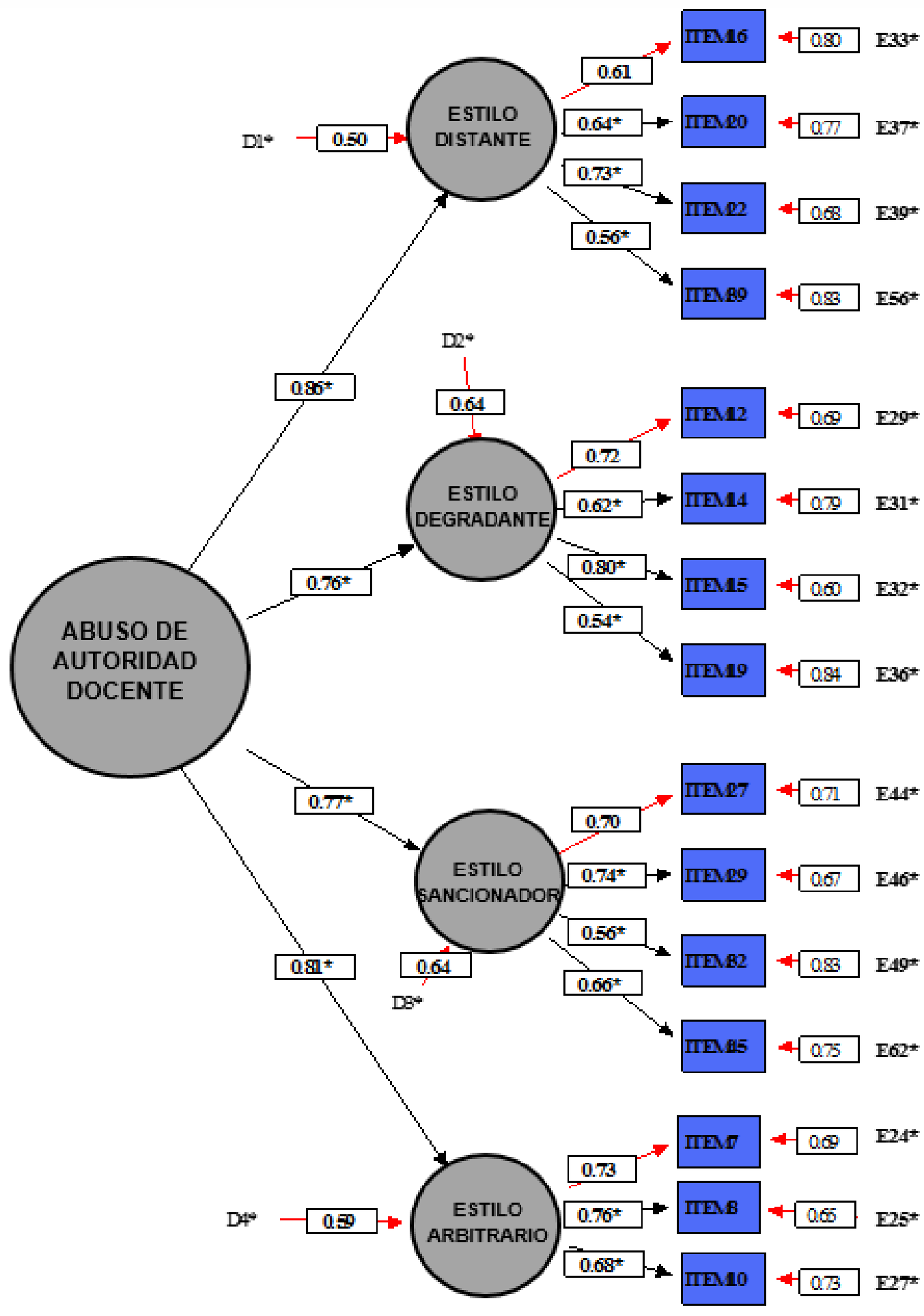

Como observamos en la tabla 3, la bondad de ajuste de este modelo apenas varía respecto al modelo anterior, siendo su Chi cuadrado de 112,55 y el $X^{2} / g l(1,32)$. Los valores GFI, AGFI, CFI, $\mathrm{NFI}, \mathrm{SRMR}$ y RMSEA se mantienen iguales a los del modelo de cuatro factores interrelacionados. Respecto a los valores de las flechas que van desde la variable latente a las dimensiones y de las dimensiones a los indicadores, los parámetros alcanzan valores altos superiores a 0,75 en el 
primer caso y a 0,55 en el segundo. Existe, por tanto, una variable exógena (abuso de autoridad docente) que explica cuatro variables intermedias o dimensiones (estilos de abuso de autoridad docente).

En cuanto a la validez externa, al observar la relación entre la puntuación total en la versión final de la EAADP y la Escala de Bienestar Personal Subjetivo (EBPS), observamos una correlación inversa $(r=-0,210)$. Este tamaño es pequeño pero significativo.

\section{Conclusiones}

El objetivo principal de este estudio era mostrar el proceso de creación y validación de una escala que permitiera a los orientadores educativos evaluar la percepción de los alumnos sobre el abuso de autoridad docente vivido durante su trayectoria escolar. En general, la EAADP resulta un instrumento válido y fiable para evaluar el Abuso de Autoridad Docente.

Los índices de consistencia interna de la escala muestran que podemos obtener unas puntuaciones con una fiabilidad aceptable sobre la percepción que tienen los alumnos del abuso de autoridad docente que han sufrido durante su etapa escolar. Por tanto, la información recogida en la EAADP permite observar diferencias estadísticamente significativas entre los alumnos en sus puntuaciones en abuso de autoridad docente. El porcentaje de alumnos que declararon haber sentido alguna vez que un profesor les trataba injustamente abusando de su autoridad durante su trayectoria escolar es similar al de otros países. El porcentaje de nuestra muestra se situó en el $70,20 \%$ frente al $64 \%$ de la muestra americana (Davies, 2011). El porcentaje es inferior al obtenido por Whitted y Dupper (2008), situado en un $88 \%$. Esta diferencia puede deberse a que en su estudio se incluyeron ítems relacionados con el maltrato físico. También difiere del porcentaje del estudio de estudiante (Twemlow et al., 2006) donde el $45 \%$ de los profesores admitieron haber acosado a un estudiante. En este caso hay que tener en cuenta que los resultados provienen de la opinión de los docentes y en nuestro estudio se trata de la vivencia subjetiva de los alumnos. Además, es necesario contemplar las variables contextuales que pueden interferir en la adaptación de la versión inglesa de la escala al castellano.

Los resultados indican que existe una relación inversa entre el abuso de autoridad docente percibido por el alumno y el bienestar personal subjetivo. Aquellos alumnos con un menor bienestar personal subjetivo son los que parecen haber sufrido un mayor abuso por parte de sus docentes. Es decir, aquellos alumnos que han sufrido más abuso de autoridad docente se sienten peor con el conjunto de su vida personal o viceversa.

La estructura de la EAADP se ha confirmado a través de un modelo de medida con unos índices de ajuste más que aceptables. Este modelo muestra cuatro variables de primer orden (estilos de abuso de autoridad docente) que permiten medir una variable latente, el Abuso de Autoridad Docente. De esta manera, no es necesario que los alumnos puntúen alto en los cuatro estilos para ser receptores de abuso, sino que podemos encontrar cuatro estilos de abuso diferentes. Los cuatro estilos de abuso de autoridad docente propuestos por el modelo son: el estilo distante, engloba aquellas conductas en las que el profesor abusa de su autoridad por omisión, es decir, evitando felicitar al alumno o preguntarle, e ignorándole dentro y fuera del aula; el estilo degradante, en el que, el profesor humilla y ridiculiza al alumno, generalmente delante de sus compañeros; el estilo sancionador, afín al uso inadecuado del castigo; y el estilo arbitrario, relacionado con la parcialidad a la hora de evaluar al alumno.

Los resultados de este estudio son similares a los obtenidos por Nesbit y Philpott (2002) en su análisis factorial en dos de estos factores, pero discrepan en la definición de los otros cuatro. Las conductas distantes y degradantes son similares a los estilos de AAD que comparten el mismo nombre. Por otro lado, el factor dominante de Nesbit y Philpott engloba conductas que humillan, 
denigran y disminuyen la autoestima mediante el bochorno (vergüenza) y el desprecio verbal. En nuestro análisis este factor ha sido denominado estilo sancionador haciendo hincapié en la forma de utilizar el castigo para controlar la conducta del alumno. Respecto al estilo arbitrario, éste se relaciona con las conductas discriminantes en tanto en cuanto perjudican a un individuo tratándolo de forma distinta a los demás, pero en el caso del estilo de abuso se centra fundamentalmente en la arbitrariedad de la evaluación. Por último, en nuestro modelo no aparecen reflejados los factores de conductas desestabilizadoras o diversas. Los expertos no identificaron estos ítems como propios del abuso de autoridad docente por tratarse de conductas más relacionadas con el abuso físico, sexual o colectivo.

Nuestro estudio presenta algunas limitaciones. En primer lugar, debemos recordar los problemas propios de las medidas de auto informe; es posible que aquellos alumnos que tienen un bajo rendimiento académico tengan una tendencia a culpar a sus profesores de sus malos resultados. Es posible que en algunos casos la disonancia cognitiva juegue un papel relevante a la hora de asumir su responsabilidad. Este hecho es especialmente relevante en el estilo arbitrario de abuso de autoridad docente. Sin embargo, como expresa Lozano (2007) "consideramos que las percepciones de la propia persona son significativas para su comportamiento y por ello debemos evaluarlas y tenerlas en cuenta" (p.346). En el caso del AAD nos interesa la vivencia del alumno.

Respecto al cuestionario en sí, destacamos la poca familiaridad de los alumnos con el formato de aplicación de la escala (plantilla de lectora óptica) y las dificultades de comprensión lectora identificadas durante el proceso. En ciertos niveles, especialmente en los cursos de diversificación, a los alumnos les costó mantener la atención durante la lectura de la escala. Este hecho puede haber afectado a la fiabilidad de sus respuestas.

La muestra es la tercera limitación de este estudio. El tipo de muestreo fue incidental y ha dependido de la colaboración del director del centro. Resultó especialmente difícil acceder a la muestra debido al tema de investigación y al estigma social que lo rodea. Así, el $50 \%$ de los centros a los que se les planteó participar en el estudio rechazaron la invitación. Es necesaria la aplicación de la escala en una muestra de mayor tamaño que incluya mayor representación en el tipo de centro (no se incluyeron privados) y diversidad territorial (solo centros de Madrid capital).

En relación con la aplicabilidad de la escala, tenemos que ser conscientes de que su uso estandarizado puede ser complicado debido a la temática que plantea. Se presenta como una prueba que permita realizar un filtro para detectar alumnos que hayan sufrido abuso de autoridad docente en su trayectoria escolar y no tanto como un instrumento diagnóstico del abuso sufrido en la actualidad. No obstante, siendo conscientes de la sensibilidad del tema, es necesario ser extremadamente prudentes a la hora de aplicar la escala en los centros.

Nuestro estudio es de una extraordinaria importancia, al constituir una primera aproximación al análisis del Abuso de Autoridad Docente percibido por los alumnos en España. No obstante, aunque hemos desarrollado una escala válida y fiable además de proponer un modelo de medida formado por cuatro variables de primer orden (estilos de abuso de autoridad docente) y una variable latente, el Abuso de Autoridad Docente, es necesario ser prudentes a la hora de extraer conclusiones a tenor de lo expuesto y tener en cuenta las limitaciones de esta investigación descritas previamente.

Como afirma Davies (2011), la escuela es una de las primeras experiencias que tiene un niño con el mundo fuera de su familia por lo que las experiencias de un niño o niña dentro de la escuela pueden configurar su autoconcepto. Por ello, es necesario que las relaciones entre alumnos y profesores sean positivas. Cuando estas relaciones no son positivas, sino que hacen sentir al alumno excluido, la autoimagen del niño puede verse afectada, tener consecuencias negativas en su autoestima (McEachern et al., 2008) y en definitiva en su bienestar personal. 
Tras nuestro trabajo, se abren nuevas líneas de investigación enfocadas a la comprensión del modelo teórico centrado en el docente. Esta línea de investigación podría apoyarse en la teoría de la desconexión moral de Bandura (2016) o en otras aproximaciones. Además. se podrían relacionar los cuatro estilos de abuso de autoridad docente con otras variables como el burnout, las competencias socioemocionales o las experiencias previas de maltrato o acoso de los profesores. De los datos de este estudio se deriva la necesidad de la creación en todos los centros educativos de un procedimiento de detección del abuso de la autoridad docente, así como de estrategias de evaluación docente (de cualquier nivel) que incluyan preguntas específicas sobre este fenómeno. Por tanto, la implicación principal que esta investigación tiene es la concienciación de la comunidad educativa y de la sociedad en general, de la existencia y prevalencia del abuso de autoridad docente en nuestras aulas, y de las cuatro dimensiones que puede presentar este abuso.

\section{Referencias Bibliográficas}

Atienza, F. L., Pons, D., Balaguer, I., y García-Merita, M. (2000). Propiedades psicométricas de la Escala de Satisfacción con la Vida en adolescentes. Psicothema, 12(2), 314-319.

Bacete, F. J., Coll, P. F., Casares, M. I. M., y Perrin, G. M. (2014). Las relaciones del profesorado con el alumnado en aulas del ciclo inicial de educación primaria. Adaptación del Questionnaire on Teacher Interaction-Early Primary (QTI-EP). Revista de Psicodidáctica, 19(1), 211-231.

Baluran, C. A., y Yap, M. A. (2013). Indicators of oppressive instruction: Verbal and non-verbal signals used by educators. International Journal of Physical and Social Sciences, 3(4), 92-110.

Bandura, A., Barbaranelli, C., Caprara, G. V., y Pastorelli, C. (1996). Mechanisms of moral disengagement in the exercise of moral agency. Journal of Personality and Social Psychology, 71(2), 364-374.

Bandura, A. (2002). Selective moral disengagement in the exercise of moral agency. Journal of Moral Education, 31(2), 101-119.

Bandura, A. (2016). Moral disengagement: How people do harm and live with themselves. New York, NY, US: Worth Publishers.

Ba-Saddik, A. S., y Hattab, A. S. (2012). Emotional abuse towards children by schoolteachers in Aden Governorate, Yemen: A cross-sectional study. BMC Public Health, 12, 1-8. doi: 10.1186/1471-2458-12-647

Brendgen, M., Bukowski, W. M., Wanner, B., Vitaro, F., y Tremblay, R. E. (2007). Verbal abuse by the teacher during childhood and academic, behavioral, and emotional adjustment in young adulthood. Journal of Educational Psychology, 99(1), 26-38. doi:10.1037/0022-0663.99.1.26

Buyse, E., Verschueren, K., Doumen, S., Van Damme, J., y Maes, F. (2008). Classroom problem behavior and teacher-child relationships in kindergarten: The moderating role of classroom climate. Journal of School Psychology, 46(4), 367-391.

Boyle, R. M. (2013). Perceptions of adult to student bullying in secondary school settings (Tesis doctoral no publicada). Marywood University: Pennsylvania.

Ceja, S., Cervantes, N., y Ramírez, L.M. (2011). Estudio de la violencia que el maestro de educación media superior ejerce sobre sus alumnos, como factor de desmotivación académica. Revista Electrónica Métodos, 1, 47-65 
Davies, S. W. (2011). Teacher bullies: A factorial analysis of perceptions of teachers' bullying behaviors (Tesis doctoral no publicada). Indiana State University: Indiana.

Díaz-Aguado, M. J., Martínez Arias, R., y Martín, J. (2010). Estudio estatal sobre la convivencia escolar en la Educación Secundaria Obligatoria. Madrid: MEPSYD

Diener, E. (2000). Subjective well - being: The science of happiness and a proposal for a national index. American Psychologist, 55, 34-43.

Diener, E., Emmons, R., Larsen, R.J., y Griffin, S. (1985). The Satisfaction with Life Scale. Journal of Personality Assessment, 49, 71-75.

Fraire, M., Longobardi, C., Prino, L. E., Sclavo, E., y Settanni, M. (2013). La escala" StudentTeacher Relationship Scale" dentro del contexto italiano: un estudio de validez factorial. Electronic Journal of Research in Educational Psychology, 11(31), 851-882.

Lozano, S. (2007). Validación de un modelo de medida de las dificultades en los procesos de toma de decisiones sobre la carrera profesional. Revista de Educación, 343, 189-190.

Lyles, S. R. B. (2014). School psychologists' experiences with teacher-to-student mistreatment. (Tesis doctoral no publicada). Liberty University: Lynchburg.

Mantilla, J. M. R., y Díaz, M. J. F. (2015). Diseño y validación de un instrumento de medida del clima en centros de educación secundaria. Educación XX1, 18(1), 71-98.

McEachern, A.G., Aluede, O., y Kenny, M.C. (2008). Emotional abuse in the classroom: Implications and interventions for counselors. Journal of Counseling and Development, 86(1), 3-10.

McEvoy, A. (2005, Septiembre 11-15). Teachers who bully students: Patterns and policy implications. Trabajo presentado en la Conference on Persistently Safe Schools: Philadelphia

Monsvold, T., Bendixen, M., Hagen, R., y Helvik, A. (2011). Exposure to teacher bullying in schools: A study of patients with personality disorders. Nordic Journal of Psychiatry, 65(5), 323-329. doi:http://dx.doi.org/10.3109/08039488.2010.546881

Mullet, J. H. (2006). The bully within us... as teachers. Academic Exchange Quarterly, 10(3), 9599.

Nesbit, W. C., y Philpott, D. F. (2002). Confronting Subtle Emotional Abuse in Classrooms. Guidance and Counseling, 17(2), 32.

Pianta, R.C., Hamre, B., y Stuhlman, M. W. (2003). Relationships between teachers and children. En W. Reynolds y G. Miller (Eds.), Comprehensive handbook of psychology (Vol. 7) Educational psychology, (pp.199-234). Hoboken, NJ: Wiley.

Sylvester, R. (2010). Teacher as bully: Knowingly or unintentionally harming students. Delta Kappa Gamma Bulletin, 77(2), 42-45.

Sharpe, G. B. (2012). Behind the closed door: Exploring teacher bullying and abuse of students, characteristics of the teacher, and impact. (Tesis inédita de doctorado). University of Toronto: Toronto.

Torre-Puente, J. C. (2016). Desconexión moral y acción docente. Padres y Maestros, 366, 34-40.

Torres, M. V. T., Infante, L., Mena, M. J. B., De la Morena, L., Raya, S., y Muñoz, A. (2006). Un cuestionario para evaluar el clima social del centro escolar. Psicothema, 18(2), 272-277.

Twemlow, S. W., y Fonagy, P. (2005). The prevalence of teachers who bully students in schools with differing levels of behavioral problems. American Journal of Psychiatry, 162(12), 23872389. 
Twemlow, S. W., Fonagy, P., Sacco, F. C., y Brethour, J. R.,Jr. (2006). Teachers who bully students: A hidden trauma. The International Journal of Social Psychiatry, 52(3), 187-198.

Weller, J. C. (2014). Abrasive teachers and principal response: A mixed-methods exploration of administrative decisions regarding teachers who bully students. (Tesis inédita de doctorado), Andrews University: Michigan

Whitted, K. S. y Dupper, D. R. (2008). Do teachers bully students? Findings from a survey of students in an alternative education setting. Education and Urban Society, 40(3), 329-341.

Fecha de entrada: 13 octubre 2016

Fecha de revisión: 1 marzo de 2018

Fecha de aceptación: 17 marzo 2018 


\section{Anexo 1: Escala de Abuso de autoridad docente inicial}

A continuación, piensa cómo ha sido tu relación con TUS PROFESORES e indica con qué frecuencia te han ocurrido estas situaciones durante tu etapa escolar.

El profesor/a...

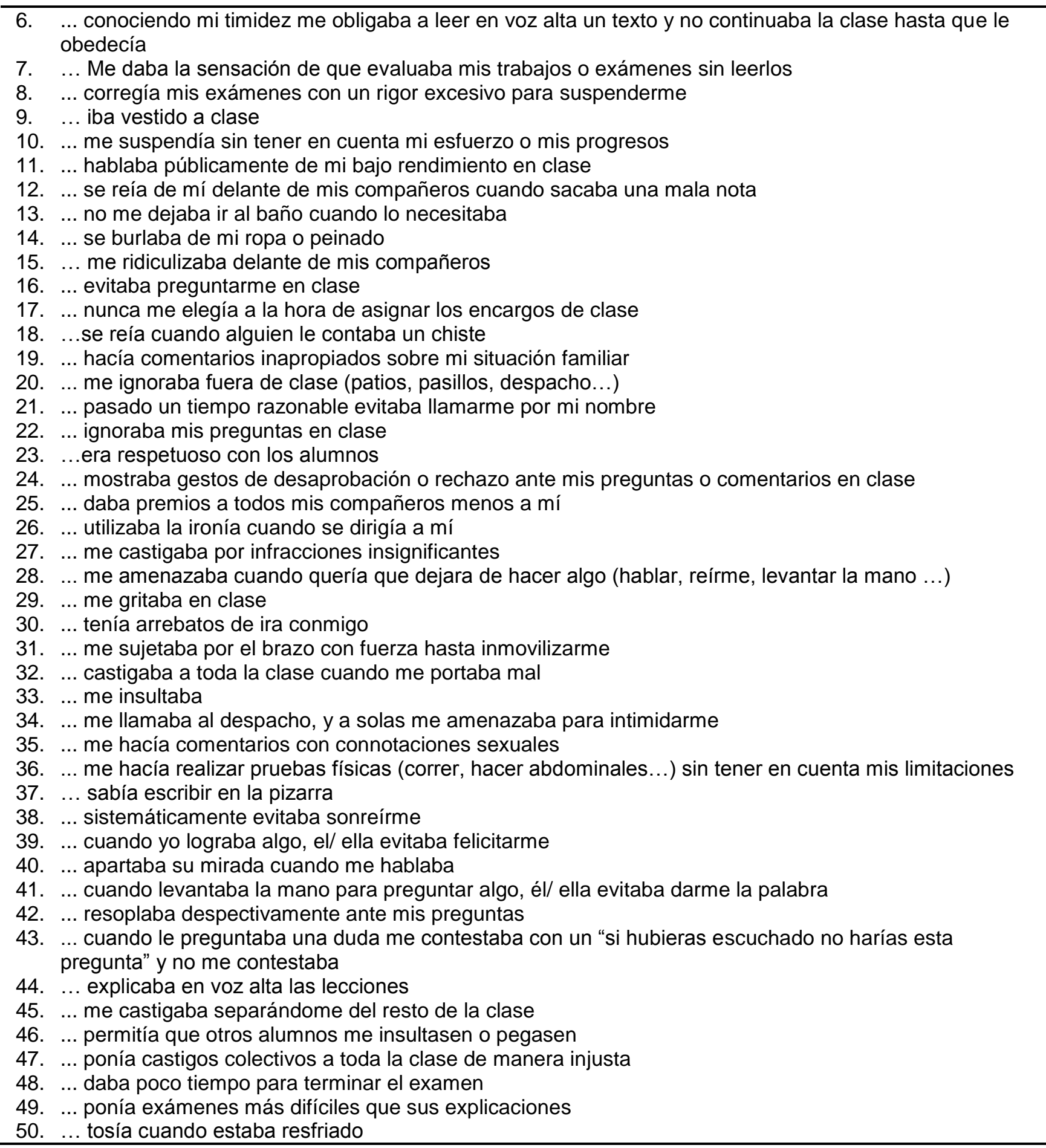

